CORRECTION

https://doi.org/10.1038/s41586-018-0530-3

\section{Author Correction: Meikin is a conserved regulator of meiosis-I- specific kinetochore function}

Jihye Kim, Kei-ichiro Ishiguro, Aya Nambu, Bungo Akiyoshi, Shihori Yokobayashi, Ayano Kagami, Tadashi Ishiguro, Alberto M. Pendas, Naoki Takeda, Yogo Sakakibara, Tomoya S. Kitajima, Yuji Tanno, Takeshi Sakuno \& Yoshinori Watanabe

Correction to: Nature https://doi.org/10.1038/nature14097, published online 22 January 2015.

In Figs. 2b, 3a and $4 c$ of this Article, oocytes were collected in several batches (each batch being the oocytes obtained from one female mouse), but statistical analysis was performed by inappropriately dividing the oocytes into three equal groups, indicated as three independent experiments (see Supplementary Fig. 1 to this Amendment). The corrected graphs, in which statistical analysis was performed among the total oocytes from pooled batches ( $n=$ oocyte number), are shown in Figs. 1, 2 and 3 of this Amendment, respectively. The corrected figure legends, including the number of oocytes, are also shown here. See Supplementary Information to this Amendment for the corrected Source Data files for Figs. 2b, $3 \mathrm{a}$ and $4 \mathrm{c}$. These corrections do not change the conclusions derived from the experiments, and the original Article has not been corrected online. We apologize for any confusion that this error may have caused.

Supplementary Information is available in the online version of this Amendment.

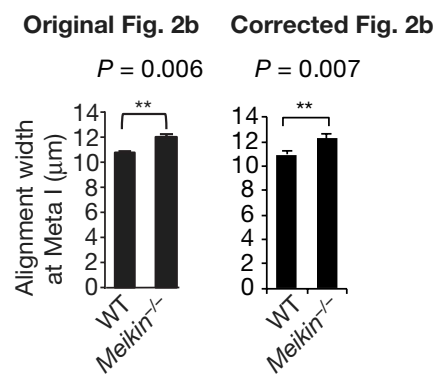

Fig. 1 | This is the corrected panel and the incorrect published panel from Fig. 2b of the original Article. Metaphase I oocytes (6 h post GVBD) stained for $\alpha$-tubulin, CENP-C and DAPI (DNA). Oocytes were collected in batches and the chromosome alignment widths were measured in each oocyte $(n=30$ oocytes, mean + s.e.m. $)$.

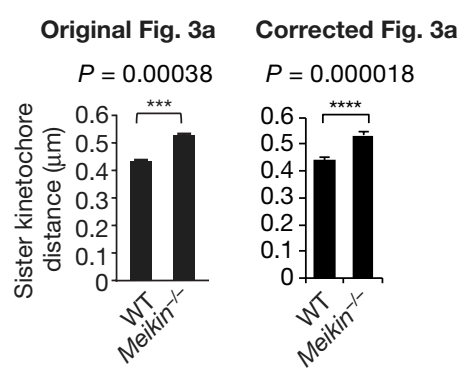

Fig. 2 This is the corrected panel and the incorrect published panel from Fig. 3a of the original Article. WT and Meikin ${ }^{-1-}$ oocytes at early prometaphase I ( $2 \mathrm{~h}$ post GVBD) stained for CENP-C and DAPI (DNA). The pairs of sister kinetochores were measured for the distance between two CENP-C signals of a sister kinetochore pair. Oocytes were collected in batches and average distance of 10 kinetochore pairs was measured in each oocyte ( $n=15$ oocytes, mean + s.e.m.).

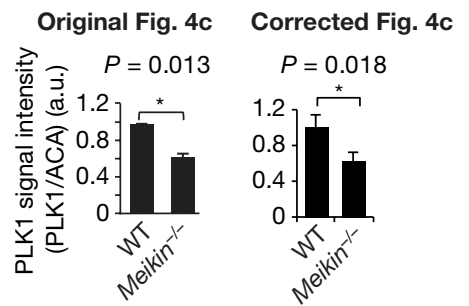

Fig. 3 This is the corrected panel and the incorrect published panel from Fig. $4 c$ of the original Article. Prometaphase I oocytes ( $3 \mathrm{~h}$ post GVBD) stained for PLK1, ACA and DAPI (DNA). Oocytes were collected in batches and average intensity of PLK1 normalized to that of ACA was measured from approximately 15 kinetochores in each oocyte $(n=12$ oocytes, mean + s.e.m.). 